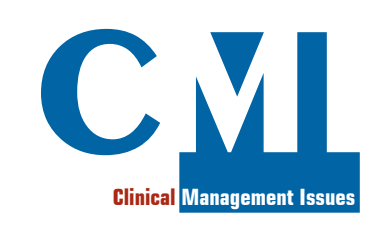

\title{
Acknowledgement to Reviewers (July 2017 - December 2018)
}

The editorial staff of Clinical Management Issues (CMI) would like to thank all the reviewers who, with their support and their active cooperation, have contributed to improving the scientific rigor, precision and accuracy of the contents.

- Giuseppe Affronti

- Jeffrey R. Andolina

- Riccardo Asteggiano

- Steven Baker

- Irene Binaco

- Giuseppe Caparrotti

- Novella Carannante

- Jose Manuel Ceresetto

- Francesco Cortese

- Roberto Dal Negro

- Gaetano D'Ambrosio

- Maria Giovanna Danieli

- Francisco Antunes Dias

- Khaled Emara

- Thierry Girard

- Giovanni Grasso

- Cristoforo Incorvaia

- Jagadeesh Kalavakunta
- George Lazaros

- Alba Lopez Bravo

- Mauro Mennuni

- Hilali Noordeen

- Giovanni Paolino

- Régis Peffault De La Tour

- Pietro Pirina

- Aurel Popa-Wagner

- Francesco Puppo

- Mohammad Robed Amin

- Andrea Rossi

- Valeria Sansone

- Jens Schmidt

- Samuel Shinjo

- Luigi Tarantini

- Ming Hsien Tsai

- Akinori Uruha

- Simone Vidale 